\title{
Vinflunine in the treatment of bladder cancer
}

\author{
Mark Bachner \\ Maria De Santis \\ 3rd Medical Department - Center \\ for Oncology and Hematology, Kaiser \\ Franz Josef-Spital der Stadt Wien, \\ and Ludwig Boltzmann-Institute \\ for Applied Cancer Research \\ Vienna (LBI-ACR VIEnna), Cluster \\ Translational Oncology, Kaiser Franz \\ Josef-Spital der Stadt Wien, and \\ Applied Cancer Research - Institution \\ for Translational Research Vienna \\ (ACR-ITR VIEnna)/CEADDP, Vienna, \\ Austria
}

\begin{abstract}
Vinflunine (VFL) is a third-generation bifluorinated semi-synthetic vinca alkaloid obtained by superacidic chemistry from its parent compound, vinorelbine. As with the other vinca alkaloids, the main antineoplastic effects of VFL arise from its interaction with tubulin, the major component of microtubules in mitotic spindles. In contrast to other vinca alkaloids, VFL shows some distinctive properties in terms of tubulin binding, possibly explaining its superior antitumor activity in vitro and in vivo compared with vinorelbine as well as its excellent safety profile. In transitional cell carcinoma (TCC), two single-agent phase II trials were performed testing VFL in platinum-pretreated patients, showing moderate response rates and promising disease control rates. Therefore, the first phase III trial in modern times for second-line TCC of the urothelium was designed in order to further investigate the activity of VFL. First results were presented at the 2008 ASCO conference. VFL appears to be a possible treatment option for patients with TCC progressing after first-line platinum-containing chemotherapy.
\end{abstract}

Keywords: vinflunine, transitional cell carcinoma (TCC) of the bladder, bladder cancer, chemotherapy, second-line chemotherapy

\section{Bladder cancer - a brief overview}

In 2002, 357,000 patients were newly diagnosed with transitional cell carcinoma of the urothelium (TCCU) worldwide, 274,000 males and 83,000 females, making it the ninth most common type of cancer for both sexes combined (Parkin et al 2005). In the same year, an estimated number of 145,000 patients died from this disease $(108,000$ males and 37,000 females).

The standard of care for muscle-invasive TCCU is radical cystectomy. Unfortunately, 5-year survival rates after surgery are only approximately 50\% (Ghoneim et al 1997; Bassi et al 1999; Dalbagni et al 2001; Stein et al 2001; Stein 2006). About $50 \%$ of patients will relapse after surgery, depending on the pathological stage of the primary tumor, the nodal status, and the quality and extent of surgery performed. Local recurrence counts for about $30 \%$ of relapses whereas distant metastases are more common (Rosenberg et al 2005).

Trying to improve outcome after surgery, multiple randomized trials examined preoperative chemotherapy, leading to inconclusive and even controversial results. Therefore, 3 meta-analyses were performed (Advanced Bladder Cancer Meta-Analysis Collaboration 2003, 2005b; Winquist et al 2004), showing a small, but statistically significant overall survival (OS) benefit for neoadjuvant, cisplatin-containing combination chemotherapy.

Five randomized trials and 1 meta-analysis could not provide convincing results to support routine use of adjuvant chemotherapy (Advanced Bladder Cancer Meta-Analysis Collaboration 2005a).

Before the development of effective chemotherapy, the median survival of patients with metastatic urothelial cancer rarely exceeded 3 to 6 months (Sternberg et al 2003). In the early 1980s, cisplatin monotherapy doubled the median survival to about 8 months (Loehrer et al 1992). MVAC (methotrexate, vinblastine, doxorubicin, 
and cisplatin) in the mid 1980s (Sternberg et al 1988), further improved OS to slightly over 1 year and became the standard of care for metastatic TCCU (Culine 2002).

In a phase III trial published in 2000, von der Maase et al (2000) established the combination regimen GC (gemcitabine, cisplatin) as an alternative to MVAC with comparable efficacy results but a favorable toxicity profile. Today, a median OS of 14 to 15 months can be expected.

For the large patient group regarded as unfit for cisplatin-containing chemotherapy (mainly due to impaired renal function and/or low performance status), there are only limited data and no approved standard of care.

Also, for patients progressing after first-line platinumcontaining chemotherapy, there is still no approved secondline therapy.

\section{Vinflunine - preclinical studies}

Vinflunine (VFL, Javlor $\left.{ }^{\circledR}\right)$ is a third-generation, semi-synthetic vinca alkaloid obtained through superacidic chemistry by the selective introduction of 2 fluorine atoms at the $20^{\prime}$-position of vinorelbine, a part of the molecule previously inaccessible by classical chemistry (Fahy et al 1997, 2008).

VFL interacts with the so-called vinca-alkaloid-bindingdomain of tubulin, as judged by proteolytic cleavage patterns (Lobert et al 1998), and, more recently, confirmed by NMR spectroscopy (Fabre et al 2002).

Microtubules are an important target for anticancer therapy because of the crucial role they play during mitosis, coordinating chromosomal segregation; microtubule inhibitors include vinca alkaloids, taxanes, and epothilones.

VFL expresses some distinctive features: the affinity of VFL binding to tubulin is considerably lower than that of other vinca alkaloids. Also, VFL does not prevent other vinca alkaloids from binding to unassembled tubulin. The binding affinity of different vinca alkaloids to tubulin was classified as: vincristine $>$ vinblastine $>$ vinorelbine $>$ vinflunine (Kruczynski et al 1998a; Lobert et al 1998), correlating well with the weekly intravenous drug doses of these vinca alkaloids used in the clinic.

Interestingly, the binding affinity of vinca alkaloids to tubulin is not necessarily related to the degree of antitumor efficacy. Singer et al (1992), for example, found an inverse correlation between relative binding affinities and inhibition of cell proliferation examining 4 different vinca alkaloids. Also, Jordan et al (1985) described that in contrast to their relative abilities to inhibit microtubule assembly in vitro, vinblastine and its derivative, vindesine, were more potent than vincristine and vinepidine in inhibiting cell proliferation in culture.
This may be due to the fact that vinca alkaloids accumulate intracellularly several-fold to $>100$-fold or that they target other intracellular sites (Gout et al 1984; Jordan et al 1991; Etievant et al 1998; Jean-Decoster et al 1999; Ngan et al 2001). Of the tested vinca alkaloids, VFL reached the highest intracellular concentrations (Hill 2001). The significance of this finding is still unclear.

Lobert et al (1998) hypothesized that the drug affinity to tubulin may contribute to the severity of neuropathies observed clinically.

Microtubules display two types of characteristic behavior: "dynamic instability", a random switching of microtubules between phases of relatively slow growth and rapid shortening and "treadmilling", a net addition of tubulin subunits at one end of a microtubule (the fast-growing plus end) and the balanced net loss from the opposite end (the slow-growing minus end). Both phenomena appear crucial for progression through mitosis and the cell cycle.

Ngan and colleagues found that the effects of vinorelbine and VFL on microtubule dynamics differ significantly from those of the classic vinca alkaloid, vinblastine (Ngan et al 2000, 2001; Jordan et al 2008): VFL and vinorelbine suppress the rate and extent of microtubule growing, whereas vinblastine strongly suppresses the rate and extent of microtubule shortening. VFL inhibited the rate of treadmilling 4-fold less strongly than vinorelbine and 7-fold less strongly than vinblastine.

Ngan et al (2000) hypothesized that non-tumor cells with "normal" checkpoint proteins could tolerate the relatively less powerful inhibitory effects of VFL and vinorelbine on microtubule dynamics rather than the more powerful effects of vinblastine, whereas tumor cells with frequently "faulty" checkpoint mechanisms may be more susceptible to VFL and vinorelbine than normal cells. This may account for the superior antitumor efficacy as well as a favorable safety profile of VFL.

VFL bound to tubulin induces structural changes favoring an inhibition of GTP hydrolysis and inhibition of microtubule assembly (Kruczynski et al 1998a; Hill 2001). In cell cultures, VFL reduced the microtubule network of interphase cells and induced G2+M arrest (Kruczynski et al 1998a), leading to mitotic accumulation at the metaphase/anaphase transition and finally resulting in apoptosis (Kruczynski et al 2002; Pourroy et al 2004; Braguer et al 2008; Jordan et al 2008). At higher concentrations, VFL - like other vinca alkaloids - aggregated microtubules, leading to paracrystal formations.

In preclinical in vivo studies, VFL showed definite (high or moderate) antitumor activity against 7/11 (64\%) of 
subcutaneously implanted human tumor xenografts compared with vinorelbine, which showed only moderate activity against 3/11 (27\%) of the xenografts (Kruczynski et al 1998b; Hill et al 1999), suggesting a broader spectrum of activity for VFL. VFL led to significant survival prolongation of tumor-bearing mice and tumor growth inhibition with optimal $\mathrm{T} / \mathrm{C}$ (treated vs control) values of up to $457 \%$ in the absence of any significant body weight loss, providing evidence of a high level of tolerance to these effective antitumor doses of VFL.

Following the clinical success of vinorelbine and its efficacy in combination with other anticancer drugs (Johnson et al 1996; Bunn et al 1998; Gregory et al 2000; Hortobagyi 2000), Barret et al (2000) studied in vitro synergistic effects of several VFL combinations in a human non-small-cell lung cancer (NSCLC) line and a human leukemia cell line. They incubated VFL with camptothecin, cisplatin, doxorubicin, etoposide, 5-fluorouracil, gemcitabine, mitomycin $\mathrm{C}$, paclitaxel, or vinorelbine. A high level of synergistic cytotoxicity of VFL was observed when combined with the DNA-damaging agents cisplatin and mitomycin $\mathrm{C}$, the DNA-intercalating agent doxorubicin, and the antimetabolite 5-fluorouracil, all of which induce DNA damage directly or indirectly and induce cell death predominantly via a p53-dependent pathway. Moderate synergy was identified with the combination of VFL and the topoisomerase I inhibitor camptothecin. Only additivity but no synergy could be shown for combinations with the topoisomerase II inhibitor etoposide, the antimetabolite gemcitabine, and either of the two tubulin-interacting agents paclitaxel and vinorelbine.

These results are promising and may implicate manifold possibilities for combination therapies including VFL. A large 3-armed study for NSCLC patients could already demonstrate significantly higher response rates (RR) for the parent compound, vinorelbine, when combined with cisplatin compared to vinorelbine alone and compared to the combination of vindesine and cisplatin (Le Chevalier et al 1994). First results of a combined phase I/II trial of VFL in combination with cisplatin - again for patients with NSCLC - were also very encouraging with a RR of $33 \%$ and a disease control rate of $77 \%$ (Ramlau et al 2004).

Following earlier studies that showed definite antivascular effects of vinblastine and vinorelbine (Baguley et al 1991; Hill et al 1993), Holwell et al studied the influence of VFL on tumor vascularization in vivo using a transplantable murine tumor model (Holwell et al 2001). Morphologic changes after VFL-treatment included extensive hemorrhagic necrosis. Perfusion studies showed a vascular shutdown over a minimum of 24 hours at doses considerably lower than the maximum tolerated dose, suggesting that VFL mediates its antitumor activities - at least in part - via an antivascular pathway (Braguer et al 2008).

Resistance of tumor cells to multiple cytotoxic drugs, termed MDR, is a major limitation to effective chemotherapy. Results of a series of studies involving both in vitro and in vivo experimental tumor models suggested that VFL, like the other vinca alkaloids, belongs to the P-glycoprotein-dependent multidrug resistant (MDR) family of anticancer agents. However, it was clearly shown that among various P-glycoprotein-overexpressing multidrug resistant human tumor sublines tested in vitro, the level of cross-resistance expressed with VFL was generally far lower than that of vinorelbine or vincristine. Testing human leukemia cells in vivo, it was demonstrated that VFL induced drug resistance far less readily than vinorelbine, both in terms of time taken for resistance to be established and the level of resistance ultimately obtained (Etievant et al 1998, 2001; Kavallaris et al 2008).

In order to investigate the feasibility of systemic treatment of TCC of the bladder with VFL, Bonfil et al (2002) examined the effect of VFL on a murine bladder cancer cell line, which was transurethrally implanted. They found clear antitumor activity of VFL against this superficial bladder cancer model superior to that of vinorelbine with a good overall tolerance, suggesting that VFL might be a good candidate for the systemic treatment of bladder cancer.

\section{Vinflunine - phase I trials}

Starting in 1998, 3 initial phase I trials with different schedules of intravenous administration were performed in order to determine the recommended dose (RD) for single agent VFL:

- day 1 in a 3-week schedule: RD at $350 \mathrm{mg} / \mathrm{m}^{2}$ every 3 weeks (Bennouna et al 2003)

- weekly administration:

- previously treated patients: RD at $120 \mathrm{mg} / \mathrm{m}^{2}$ (Delord et al 2001; Puozzo et al 2001)

- previously untreated patients: RD at $150 \mathrm{mg} / \mathrm{m}^{2}$ (Vermorken et al 2003)

- day 1 and day 8 every 3 weeks: RD at $170 \mathrm{mg} / \mathrm{m}^{2}$ (Zorza et al 2001; Johnson et al 2001, 2006)

Dose-limiting toxicities (DLT) in these classical single agent phase I trials included grade 4 neutropenia, febrile neutropenia, grade 3/4 constipation, grade 3 myalgia, 
grade 3 chest pain, grade 4 infection, and grade 3 rise in transaminases.

The VFL dose and schedule selected for phase II studies were 1 intravenous administration every 3 weeks at $350 \mathrm{mg} / \mathrm{m}^{2}$ (Bennouna et al 2003), since the other schedules did not result in a higher dose intensity. However, after an analysis of data from the first 24 patients enrolled in phase II trials, the initial RD was lowered to $320 \mathrm{mg} / \mathrm{m}^{2}$ every 3 weeks in patients with good performance status (PS) and no prior extended pelvic irradiation, and to $280 \mathrm{mg} / \mathrm{m}^{2}$ for other patients.

In 2002, Focan et al (2002) presented pharmacokinetic results of five patients treated with radiolabeled, tritiated VFL given iv at $250 \mathrm{mg} / \mathrm{m}^{2}$. They described 11 metabolites of VFL, the predominant and only active one being 4-Odeacetyl-vinflunine (DVFL). Two thirds of the dose was eliminated through bile and one third by the kidneys (Focan et al 2002; Lobert et al 2008).

Different phase I trials with VFL in combination with other anticancer drugs were performed or are still ongoing, including pemetrexed (Shah et al 2008), trastuzumab (Paridaens et al 2007), carboplatin (Tourani et al 2005), gemcitabine (Lemarie et al 2005), cisplatin (Ramlau et al 2004), capecitabine, erlotinib, and cetuximab (www.clinicaltrials.gov).

Paule et al (2007) performed a phase I trial in 30 patients to determine VFL dose adjustments in cancer patients with various degrees of liver dysfunction. They found that pharmacokinetic parameters of VFL and DVFL did not appear to be affected by the degree of liver disease. Saliba et al (2007) presented a subgroup analysis of the 18 patients with hepatocellular carcinoma taking part in the above mentioned trial and concluded that VFL could be given safely at $320 \mathrm{mg} / \mathrm{m}^{2}$ in this patient cohort. The disease control rate was promising (66.7\%) with one partial response (PR).

At the 2008 ASCO annual meeting Bennouna et al (2008) presented preliminary data of a phase I trial with oral VFL given twice a day for 2 consecutive days every week. The bioavailability of this oral form was $57 \%$, the maximum tolerated dose was reached at $300 \mathrm{mg} /$ day, when 2 patients experienced febrile neutropenia concomitant with grade 3 diarrhea. The recommended dose for oral application has not yet been defined.

\section{Vinflunine in bladder cancer - phase II trials}

In 2006, Culine et al published results of a phase II study of VFL as a 10-minute infusion 3-weekly in bladder cancer patients failing or progressing after first-line platinumcontaining chemotherapy or after platinum-containing regimens given with adjuvant or neoadjuvant intent (Culine et al 2006). Fifty-eight patients were recruited in this multicenter trial by 16 European centers between November 2000 and September 2002. The primary objective was overall response rate (ORR), secondary objectives were duration of response, progression-free survival (PFS), OS, and safety. Eligibility criteria included a Karnofsky performance status (KPS) of 80 or greater and a glomerular filtration rate of at least $40 \mathrm{~mL} / \mathrm{min}$.

One patient died before receiving treatment and was not included in the analysis. At the beginning of the study, 6 patients were treated with intravenous (iv) VFL at $350 \mathrm{mg} / \mathrm{m}^{2}$ based on the above described phase I trial (Bennouna et al 2003). The most frequent adverse events at this dose included leucopenia, neutropenia, and anemia, which were observed in all 6 patients $(100 \%)$ with 1 fatal febrile neutropenia. A preliminary safety evaluation, performed across all ongoing VFL phase II trials, led to a dose reduction to $320 \mathrm{mg} / \mathrm{m}^{2}$ 3-weekly (the next lower dose level of the preceding phase I trial). The 6 patients treated at $350 \mathrm{mg} / \mathrm{m}^{2}$ were not included in the analysis.

The median age of the analyzed 51 patients was 63 years; $55 \%$ had a KPS of 100 or 90 . As prior chemotherapies, 22 patients (43\%) had received MVAC or CMV (cisplatin, methotrexat, vinblastin) and 25 patients (49\%) GC; prior therapy was for advanced disease in 34 patients $(67 \%)$ and as adjuvant or neoadjuvant chemotherapy in 17 patients (33\%). The median treatment-free interval between completion of initial chemotherapy and VFL treatment was 7.5 months. All patients enrolled in the study had clear evidence of progressive disease (PD), $61 \%$ had 2 or more metastatic lesions at entry, and $49 \%$ had visceral involvement.

There were 9 PR observed (18\%), 25 patients had stable disease (SD), amounting to a disease control rate of $67 \%$.

Disease control rates seemed to correlate with the interval from prior platinum treatment, with better results in late relapsing or progressing patients. ORR were $8 / 34$ $(24 \%)$ and $1 / 17(6 \%)$ in patients previously treated in the metastatic and neoadjuvant/adjuvant setting, respectively. Responses were observed in 3/22 (14\%) patients who had received prior vinca alkaloids as a part of the MVAC or CMV regimens. Responses were predominantly seen in patients who had previously responded to chemotherapy, although numbers were too small to exclude random variation. However, 5 out of $25(20 \%)$ of patients with visceral involvement achieved an objective response and responses were also seen in patients with primary chemoresistant disease. 
Median duration of response was 9.1 months. Among the 51 patients treated at $320 \mathrm{mg} / \mathrm{m}^{2}$, median PFS was 3.0 months and median OS was 6.6 months.

During treatment, KPS improved in 11 patients $(22 \%)$, 27 patients $(53 \%)$ maintained their baseline status. Only 10 patients $(20 \%)$ had a worsening of their KPS during treatment.

Toxicity was generally well manageable and noncumulative; the predominant grade $3 / 4$ hematological toxicity was neutropenia (67\%), with 5 patients $(10 \%)$ experiencing febrile neutropenia, 2 of whom died; both had received multiple courses of VFL. The main grade 3/4 non-hematologic toxicities included fatigue (10\%), constipation ( $8 \%$ ) and abdominal pain $(8 \%)$. Of note, there was no grade 3 or 4 peripheral neurotoxicity observed and no grade $3 / 4$ rise in serum creatinine.

At the 2008 ASCO Genitourinary Cancers Symposium, Vaughn et al (2008) presented results of a second international phase 2 trial conducted to confirm the results published by Culine et al (2006). Eligibility criteria were comparable: patients with no more than one prior platinum-based regimen with disease progression within 12 months of treatment, a KPS $\geq 80$, and a creatinine clearance $>20 \mathrm{~mL} / \mathrm{min}$. The primary endpoint was ORR. 175 patients were enrolled, of whom 151 received treatment and were included in the analysis. VFL $320 \mathrm{mg} / \mathrm{m}^{2}$ was administered once every 3 weeks as a 15- to 20-minute intravenous infusion. Patients with KPS 90 or 80 , prior pelvic irradiation, over 75 years of age, or a creatinine clearance between 20 and $60 \mathrm{~mL} / \mathrm{min}$ received an initial dose of $280 \mathrm{mg} / \mathrm{m}^{2}$, which was escalated to $320 \mathrm{mg} / \mathrm{m}^{2}$ from cycle two onwards, based on tolerance.

Twenty-two PR with a median duration of 6.0 months were reported, equivalent to an ORR of $14.6 \%$. Sixty-four patients $(42.4 \%)$ had SD with a median duration of 4.0 months, resulting in a disease control rate of $57.0 \%$. Median PFS was 2.8 months and median OS was 7.9 months.

Toxicity was similar to the data previously reported by Culine (2006), with $58.1 \%$ of patients experiencing neutropenia grade $3 / 4$ and 10 patients $(6.6 \%)$ with neutropenic fever. Grade 3/4 non-hematologic toxicities included constipation (16.6\%), asthenia/fatigue (12.6\%), ileus (4.6\%), and abdominal pain (4.6\%).

Table 1 summarizes adverse events seen in more than 1000 patients treated with VFL as a single agent. Table 2 summarizes clinical trials with single-agent second-line chemotherapy in TCCU patients. Table 3 summarizes clinical trials with second-line combination chemotherapy.

Table I Selected adverse events in $\geq 1 \%$ of patients $(n=1049)$ (derived from Bellmunt et al 2008)

\begin{tabular}{|c|c|c|c|}
\hline & $\begin{array}{l}\text { Overall incidence } \\
\text { n (\%) }\end{array}$ & $\begin{array}{l}\text { Grade } 3 \\
\text { n (\%) }\end{array}$ & $\begin{array}{l}\text { Grade } 4 \\
\text { n (\%) }\end{array}$ \\
\hline \multicolumn{4}{|l|}{ Hematologic } \\
\hline Anemia & $787(84)$ & $82(8)$ & $14(\mathrm{I})$ \\
\hline Leukopenia & $773(74)$ & $231(22)$ & $12 \mid(\mid 2)$ \\
\hline Neutropenia & $758(72)$ & $222(21)$ & $288(27)$ \\
\hline Thrombocytopenia & $395(38)$ & $30(3)$ & - \\
\hline Febrile neutropenia & $59(6)$ & $52(5)$ & - \\
\hline Neutropenia with infection & $19(2)$ & $14(1)$ & - \\
\hline \multicolumn{4}{|l|}{ Non-hematologic } \\
\hline Abdominal pain & $255(24)$ & $56(5)$ & - \\
\hline Constipation & $552(53)$ & $108(10)$ & - \\
\hline Diarrhea & $116(11)$ & $8(1)$ & - \\
\hline Nausea & $435(4 I)$ & $30(3)$ & - \\
\hline Vomiting & $313(30)$ & $32(3)$ & - \\
\hline Stomatitis & 351 (33) & $16(2)$ & - \\
\hline Fatigue & $566(54)$ & $128(12)$ & - \\
\hline Myalgia & $195(19)$ & $32(3)$ & - \\
\hline Peripheral sensory neuropathy ${ }^{a}$ & $39(4)$ & - & - \\
\hline Injection-site reactions & $100(10)$ & - & - \\
\hline Alopecia & 307 (29) & - & - \\
\hline
\end{tabular}

A total of 880 patients were administered an initial dose of $320 \mathrm{mg} / \mathrm{m}^{2}, 169$ patients were administered an initial dose of $280 \mathrm{mg} / \mathrm{m}^{2}$. ${ }^{a}$ one case of grade 3 paresthesia $(0.1 \%)$ has been reported. 
Table 2 Single-agent second-line chemotherapies for advanced bladder cancer

\begin{tabular}{|c|c|c|c|c|c|}
\hline Trial & Regimen & $\mathbf{N}$ (evaluable) & $\mathbf{R R}$ & TTP & $\begin{array}{l}\text { Median survival } \\
\text { (months) }\end{array}$ \\
\hline (Khorsand et al I997) & Piritrexim & $17(13)$ & $23 \%$ & NR & NR \\
\hline (McCaffrey et al I997) & Docetaxel & $31(30)$ & $13 \%$ & NR & 9 \\
\hline (Papamichael et al I997) & Paclitaxel & $14(14)$ & $7 \%$ & NR & NR \\
\hline (Pronzato et al 1997) & Ifosfamide & $20(20)$ & $5 \%$ & 6 & 8 \\
\hline (Witte et al 1997) & Ifosfamide & $60(56)$ & $20 \%$ & 2.2 & 5.1 \\
\hline (Lorusso et al 1998) & Gemcitabine & $35(31)$ & $23 \%$ & 3.8 & 5 \\
\hline (Witte et al 1998) & Topotecan & $46(44)$ & $9 \%$ & 1.4 & 6.3 \\
\hline (Gebbia et al I999) & Gemcitabine & $24(24)$ & $29 \%$ & NR & 13.0 \\
\hline (Dodd et al 2000) & Pyrazoloacridine & $14(14)$ & $0 \%$ & NR & 9 \\
\hline (Albers et al 2002) & Gemcitabine & $30(28)$ & $11 \%$ & 4.9 & 8.7 \\
\hline (Roth et al 2002) & Piritrexim & $35(27)$ & $7 \%$ & 2.1 & 7.0 \\
\hline (Vaughn et al 2002) & Paclitaxel & $3 I(3 \mid)$ & $10 \%$ & 2.2 & 7.2 \\
\hline (Moore et al 2003) & Oxaliplatin & $20(18)$ & $6 \%$ & NR & NR \\
\hline (Joly et al 2004) & Paclitaxel & $45(37)$ & $5 \%$ & NR & NR \\
\hline (Sridhar et al 2005) & Bortezomib & $18(11)$ & $0 \%$ & NR & NR \\
\hline (Wülfing et al 2005) & Lapatinib & $59(59)$ & $2 \%$ & 2.0 & 4.2 \\
\hline (Sweeney et al 2006) & Pemetrexed & $47(47)$ & $28 \%$ & 2.9 & 9.6 \\
\hline (Culine et al 2006) & VFL & $58(5 I)$ & $18 \%$ & 3.0 (PFS) & 6.6 \\
\hline (Dreicer et al 2007) & Epothilone B & $45(42)$ & $12 \%$ & 2.7 (PFS) & 8 \\
\hline (Galsky et al 2007) & Pemetrexed & $13(12)$ & $8 \%$ & NR & NR \\
\hline (Vaughn et al 2008) & VFL & $175(151)$ & $15 \%$ & 2.8 (PFS) & 7.9 \\
\hline (Bellmunt Molins et al 2008) & VFL & $253(185)$ & $9 \%$ & 3.0 (PFS) & NR \\
\hline
\end{tabular}

Abbreviations: RR, response rate;TTP, time to progression; NR, not reported; PFS, progression-free survival.

\section{Second-line TCCU treatment - interpretation of trial results and open questions}

For more than 20 years, cisplatin combination regimens have been the standard of care in the treatment of locally advanced or metastatic TCCU. Urothelial cancers are highly responsive to chemotherapy in the first-line setting with possible complete responses and the potential for long-term survival. Median survival of patients has been reported to be up to 15 months. Combination regimens have demonstrated a clear advantage compared to single-agent therapy.

However, cures are rare and therapeutic options for patients refractory to or relapsing after platinum-containing chemotherapy are clearly needed. Although there were several phase II studies performed in patients with recurrent TCC, there is still no approved treatment option in this setting and no therapy has proven to prolong survival.

There are no established predictive or prognostic factors for second-line treatment in TCCU. In a retrospective multivariate regression analysis performed by Bajorin et al (1999), the authors found a KPS less than $80 \%$ and the presence of visceral metastases to be two independent prognostic factors for survival and assigned patients to three risk categories according to the number of unfavorable characteristics. In this retrospective analysis, all but 12 patients were chemonaïve; whether these prognostic factors are also valid in second-line treatment remains unclear.

Response to second-line therapy might be influenced by chemosensitivity to first-line treatment (Albers et al 2002), PS (Meluch et al 2001), the presence of visceral metastases, the intent of prior treatment (perioperative vs metastatic), or a combination of these factors (Sternberg et al 2001).

Repeatedly, promising phase II results could not be confirmed by other investigators. For example, in 1992 Logothetis et al (1992) reported a response rate of $61 \%$ for the combination of 5-FU, $\alpha$-interferon and cisplatin in partly heavily pretreated patients. Unfortunately, a subsequent EORTC trial was unable to confirm these results, reporting a RR of only $12.5 \%$ (De Mulder et al 2000). This emphasizes that initial encouraging results of small phase II trials should be confirmed by other investigators. 
Table 3 Second-line chemotherapies for advanced bladder cancer

\begin{tabular}{|c|c|c|c|c|}
\hline Trial & Regimen & $\mathbf{N}$ (evaluable) & $\mathbf{R R}$ & $\begin{array}{l}\text { Median survival } \\
\text { (months) }\end{array}$ \\
\hline (Logothetis et al 1992) & 5-FU/ $\alpha$-interferon/cisplatin & 28 (NR) & $61 \%$ & NR \\
\hline (Tu et al 1995) & Paclitaxel/methotrexate/cisplatin & $25(25)$ & $40 \%$ & NR \\
\hline (Sweeney et al 1999) & Paclitaxel/ifosfamide & $13(13)$ & $15 \%$ & 8 \\
\hline (De Mulder et al 2000) & 5-FU/ $\alpha$-interferon/cisplatin & $43(40)$ & $13 \%$ & 4.9 \\
\hline (Kaufman et al 2000) & Gemcitabine/paclitaxel & $6(6)$ & $0 \%$ & NR \\
\hline (Krege et al 200I) & Docetaxel/ifosfamide & $22(20)$ & $25 \%$ & 4 \\
\hline (Meluch et al 200I) & Gemcitabine/paclitaxel & $15(15)$ & $47 \%$ & NR \\
\hline (Sternberg et al 200I) & Gemcitabine/paclitaxel & $41(40)$ & $60 \%$ & 14.4 \\
\hline (Bellmunt et al 2002) & Methotrexate/paclitaxel & $20(19)$ & $32 \%$ & 5 \\
\hline (Pagliaro et al 2002) & Cisplatin/gemcitabine/ifosfamide & $51(49)$ & $41 \%$ & 9.5 \\
\hline (Chen et al 2004) & Gemcitabine/docetaxel/carboplatin & NR (9) & $56 \%$ & NR \\
\hline (Vaishampayan et al 2005) & Carboplatin/paclitaxel & $44(44)$ & $16 \%^{\mathrm{a}}$ & 6 \\
\hline (Fechner et al 2006) & Gemcitabine/paclitaxel & $30(27)$ & $44 \%$ & NR \\
\hline (Lin et al 2007) & Gemcitabine/ifosfamide & $23(23)$ & $22 \%$ & 4.8 \\
\hline (Albers et al 2008) & Gemcitabine/paclitaxel & $51(29)$ & $35 \%$ & 7.5 \\
\hline
\end{tabular}

alncluding 4 unconfirmed PR.

Abbreviations: RR, response rate; TTP, time to progression; NR, not reported; PFS, progression-free survival.

As Sweeney pointed out elsewhere (Sweeney et al 2006), variability in RR reported in phase II trials is not only likely to be due to a variability in drug activity, but also to the confounding factor of differing patient populations between studies. For example, many trials in second-line TCC not only include patients after first-line therapy for metastatic disease, but also allow patients having relapsed after chemotherapy in the perioperative setting. In view of this, an exact description of the patient characteristics becomes critical to evaluate the results of clinical trials.

It has been a common understanding that patients after neoadjuvant or adjuvant chemotherapy would have a higher chance of response to second-line treatment than patients relapsing after first-line MVAC for metastatic disease. For example, Sternberg et al (2001) reported RR of $80 \%$ and $27 \%$ for these two patient groups, respectively.

Interestingly, other investigators reported comparable or even worse results for patients included in second-line studies after failure of neoadjuvant or adjuvant therapy compared to patients at relapse after first-line chemotherapy (Culine et al 2006; Sweeney et al 2006).

\section{Vinflunine in bladder cancer - phase III trial}

At the 2008 ASCO conference in Chicago, Bellmunt Molins et al (2008) presented data from a multicenter, randomized
(2:1) phase III trial comparing VFL and best supportive care (BSC) (arm A) versus BSC alone (arm B) in platinumpretreated patients. In a large international effort, 83 centers from 21 countries enrolled 370 patients between May 2003 and August 2006 with unresectable locally advanced or metastasized TCCU, making this the first second-line phase III trial in "modern" times.

The primary endpoint was OS; secondary endpoints included PFS, RR, disease control, clinical benefit, and quality of life (QoL). Stratification factors were center and refractory disease (PD within 2 cycles). "Moderate neuropathy" was an exclusion criterion.

VFL was administered intravenously at a dose of $320 \mathrm{mg} / \mathrm{m}^{2}$ every 3 weeks, except for patients with a PS of 1 and/or previous pelvic irradiation, who started at $280 \mathrm{mg} / \mathrm{m}^{2}$ with a subsequent dose escalation to $320 \mathrm{mg} / \mathrm{m}^{2}$, where possible.

The statistical hypothesis was to demonstrate an OS benefit of 2 months in the VFL group (6 vs 4 months). Main characteristics of the patients were well balanced except for PS, that slightly favored arm B (PS 1 arm A 71.5\%, arm B $61.5 \%)$. Fifty-three percent of the patients were under 65 years of age and only patients in PS 0 or 1 were eligible; on the other hand, $40 \%$ of patients had bulky disease, $74 \%$ suffered from visceral involvement and over $80 \%$ of the patients had relapsed or progressed within 6 months after first-line platinum-containing chemotherapy. 
In the VFL arm a high incidence of neutropenia grade $3 / 4$ was observed $(50 \%)$, but only $6 \%$ of patients suffered from febrile neutropenia. There was one toxic death. Grade 3/4 non-hematological toxicities are summarized in Table 4.

Analyzing the intent-to-treat-population, the 2-month survival advantage for arm A was achieved (6.9 vs 4.6 months), but did not reach statistical significance ( $p$ value 0.29). In a preplanned analysis looking only at eligible/per protocol patients (13 patients not eligible, 19 patients not treated according to protocol), median OS was 6.9 months in the VFL arm and 4.3 months in the BSC arm ( $\mathrm{p}$ value 0.04 for eligible patients and 0.02 for per protocol patients). A planned multivariate analysis adjusting for prognostic factors also showed a statistically significant effect of VFL on OS ( $p=0.04)$, although other factors such as hemoglobinlevel, visceral involvement or PS had a stronger impact on survival in this analysis than the treatment with VFL.

ORR in the VFL-arm was $8.6 \%$ and therefore clearly lower than in the previous phase II trials, disease control rate was $41.1 \%$ and PFS was 3.0 months, all reaching statistical significance with $\mathrm{p}$ values $<0.01$ compared to the control arm. Despite the low response rate those responses were durable, as the median duration of response was 7.4 months, the median duration of disease control was 5.7 months.

The median duration of treatment in the VFL-arm was 9.5 weeks, roughly amounting to the survival benefit shown. As the QoL results were not yet reported, the QoL-adjusted benefit for patients treated in the VFL arm remains to be seen.

Although there is no approved standard for second-line treatment and BSC was therefore chosen as control arm in this phase III trial, several other substances have proved activity in phase II trials as well as in daily clinical routine for this patient group (see Tables 2 and 3).

\section{Conclusion}

VFL showed very promising preclinical results with a broad spectrum of activity consistently superior to that of its parent compound, vinorelbine.

Phase I trials recommended a dose of $350 \mathrm{mg} / \mathrm{m}^{2}$ as a short iv infusion on day 1 of a 3 -week cycle (Bennouna et al 2003), later reduced to $320 \mathrm{mg} / \mathrm{m}^{2}$.

In TCCU, VFL showed moderate activity in 2 phase II trials. In a large phase III trial, no statistically significant benefit was found for VFL in an intention to treat analysis; however, a preplanned secondary analysis did find an improvement in OS for patients in the VFL arm.

VFL has an excellent safety profile, the most common side effects being myelosuppression and constipation. Of note, VFL is far less neurotoxic in comparison to other vinca alkaloids and other microtubule inhibitors such as taxanes and epothilones. Therefore, VFL appears to be a reasonable option for patients with TCCU progressing after first-line platinum-containing chemotherapy.

However, treatment within clinical trials should be first choice whenever possible, in order to identify more efficient treatment options for this patient group.

Future indications for VFL in TCCU might include patients ineligible for first-line treatment with platinum-containing combination regimens. A trial comparing VFL plus gemcitabine versus gemcitabine alone has been initiated in the United States.

The role of VFL in NSCLC and metastatic breast cancer is under investigation.

Table 4 Non-hematological toxicities, $n=248$ patients (derived from Bellmunt Molins et al 2008)

\begin{tabular}{|c|c|c|c|c|}
\hline \multirow[t]{2}{*}{ Adverse event } & \multicolumn{2}{|l|}{ VFL + BSC } & \multicolumn{2}{|l|}{ BSC } \\
\hline & $\begin{array}{l}\text { All grades } \\
\text { n (\%) }\end{array}$ & $\begin{array}{l}\text { Grade } 3 / 4 \\
\text { n (\%) }\end{array}$ & $\begin{array}{l}\text { All grades } \\
\text { n (\%) }\end{array}$ & $\begin{array}{l}\text { Grade } 3 / 4 \\
\text { n (\%) }\end{array}$ \\
\hline Nausea & $97(39.1)$ & $6(2.4)$ & $25(21.4)$ & I (0.9) \\
\hline Vomiting & $72(29.0)$ & $7(2.8)$ & $17(14.5)$ & 0 \\
\hline Abdominal pain & $39(15.7)$ & $10(4.0)$ & $21(17.9)$ & $7(6.0)$ \\
\hline Constipation & I I 8 (47.6) & $40(16.1)$ & $29(24.8)$ & I (0.9) \\
\hline Stomatitis/mucositis & $7 \mid(28.6)$ & $4(1.6)$ & $2(1.7)$ & 0 \\
\hline Fatigue/asthenia & $124(50.0)$ & $48(19.3)$ & $71(60.7)$ & $21(17.9)$ \\
\hline Myalgia & $40(16.1)$ & $8(3.2)$ & $8(6.8)$ & 0 \\
\hline Neuropathy sensory & $30(12.1)$ & $3(1.2)$ & I3 (II.I) & 0 \\
\hline Alopecia & $72(29.0)$ & - & $2(1.7)$ & 0 \\
\hline Infusion site reaction & $68(27.4)$ & $\mathrm{I}(0.4)$ & 0 & 0 \\
\hline
\end{tabular}

Abbreviations: BSC, best supportive care; VFL, vinflunine. 


\section{Disclosures}

Neither author has conflicts of interest to disclose.

\section{References}

Advanced Bladder Cancer Meta-analysis Collaboration. 2003. Neoadjuvant chemotherapy in invasive bladder cancer: a systematic review and meta-analysis. Lancet, 361:1927-34.

Advanced Bladder Cancer Meta-analysis Collaboration. 2005a. Adjuvant chemotherapy in invasive bladder cancer: a systematic review and meta-analysis of individual patient data Advanced Bladder Cancer (ABC) Meta-analysis Collaboration. Eur Urol, 48:189-99; discussion 199-201.

Advanced Bladder Cancer Meta-analysis Collaboration. 2005b. Neoadjuvant chemotherapy in invasive bladder cancer: update of a systematic review and meta-analysis of individual patient data advanced bladder cancer (ABC) meta-analysis collaboration. Eur Urol, 48:202-5; discussion 205-6.

Albers P, Siener R, Hartlein M, et al. 2002. Gemcitabine monotherapy as second-line treatment in cisplatin-refractory transitional cell carcinoma - prognostic factors for response and improvement of quality of life. Onkologie, 25:47-52.

Albers P, Siener R, Park S, et al. 2008. Randomized phase III trial of 2nd line gemcitabine/paclitaxel chemotherapy in patients with advanced bladder cancer: Temporary versus maintenance treatment (German Association of Urologic Oncology (AUO) Trial AB 20/99). J Clin Oncol, 26(20 Suppl):Abstr 5030.

Baguley BC, Holdaway KM, Thomsen LL, et al. 1991. Inhibition of growth of colon 38 adenocarcinoma by vinblastine and colchicine: evidence for a vascular mechanism. Eur J Cancer, 27:482-7.

Bajorin DF, Dodd PM, Mazumdar M, et al. 1999. Long-term survival in metastatic transitional-cell carcinoma and prognostic factors predicting outcome of therapy. J Clin Oncol, 17:3173-81.

Barret JM, Etievant C, Hill BT. 2000. In vitro synergistic effects of vinflunine, a novel fluorinated vinca alkaloid, in combination with other anticancer drugs. Cancer Chemother Pharmacol, 45:471-6.

Bassi P, Ferrante GD, Piazza N, et al. 1999. Prognostic factors of outcome after radical cystectomy for bladder cancer: a retrospective study of a homogeneous patient cohort. J Urol, 161:1494-7.

Bellmunt J, Cos J, Cleries R, et al. 2002. Feasibility trial of methotrexatepaclitaxel as a second line therapy in advanced urothelial cancer. Cancer Invest, 20:673-85.

Bellmunt J, Delgado FM, George C. 2008. Clinical Activity of Vinflunine in Transitional Cell Carcinoma of the Urothelium and Other Solid Tumors. Semin Oncol, 35: S34-S43.

Bellmunt Molins J, von der Maase H, Theodore C, et al. 2008. Randomised phase III trial of vinflunine (V) plus best supportive care (B) vs B alone as 2 nd line therapy after a platinum-containing regimen in advanced transitional cell carcinoma of the urothelium (TCCU). J Clin Oncol, 26(20 Suppl):Abstr 5028.

Bennouna J, Fumoleau P, Armand JP, et al. 2003. Phase I and pharmacokinetic study of the new vinca alkaloid vinflunine administered as a 10-min infusion every 3 weeks in patients with advanced solid tumours. Ann Oncol, 14:630-7.

Bennouna J, Vermorken JB, Senellart H, et al. 2008. Phase I dose-escalation study of oral vinflunine given twice a day for 2 consecutive days every week in patients with solid tumours. J Clin Oncol, 26(20 Suppl):Abstr 13555).

Bonfil RD, Russo DM, Binda MM, et al. 2002. Higher antitumor activity of vinflunine than vinorelbine against an orthotopic murine model of transitional cell carcinoma of the bladder. Urol Oncol, 7:159-66.

Braguer D, Barret JM, McDaid H, et al. 2008. Antitumor Activity of Vinflunine: Effector Pathways and Potential for Synergies. Semin Oncol, 35:S13-S21.

Bunn PA Jr, Kelly K. 1998. New chemotherapeutic agents prolong survival and improve quality of life in non-small cell lung cancer: a review of the literature and future directions. Clin Cancer Res, $4: 1087-100$.
Chen AC, Hovey E, Shelton G, et al. 2004. Phase I/II study of docetaxel (D), gemcitabine (G), Carboplatin (C) in poor prognosis and previously treated patients (pts) with urothelial carcinoma (UTC). J Clin Oncol, 22; 2004. ASCO Annual Meeting Proceedings (Post-Meeting Edition) 14S (July 15 Supplement): 4580.

Culine S, Theodore C, De Santis M, et al. 2006. A phase II study of vinflunine in bladder cancer patients progressing after first-line platinum-containing regimen. Br J Cancer, 94:1395-401.

Culine S. 2002. The present and future of combination chemotherapy in bladder cancer. Semin Oncol, 29: 32-9.

Dalbagni G, Genega E, Hashibe M, et al. 2001. Cystectomy for bladder cancer: a contemporary series. $J$ Urol, 165:1111-6.

De Mulder PH, Theodore C, Sella A, et al. 2000. Phase II EORTC trial with 5-fluorouracil, cisplatin and interferon-alpha as second-line treatment of advanced transitional cell cancer of the urothelial tract. Ann Oncol, $11: 1391-4$.

Delord JP, Stupp R, Pinel MC, et al. 2001. Phase I Study of vinflunine given as a 10 minute intravenous (iv) Infusion on a weekly schedule in patients (pts) with advanced solid tumours. Proc Am Soc Clin Oncol, 20:2001 (Abstr 441).

Dodd PM, McCaffrey JA, Mazumdar M, et al. 2000. Phase II trial of pyrazoloacridine as second-line therapy for patients with unresectable or metastatic transitional cell carcinoma. Invest New Drugs, 18:247-51.

Dreicer R, Li S, Manola J, et al. 2007. Phase 2 trial of epothilone B analog BMS-247550 (ixabepilone) in advanced carcinoma of the urothelium (E3800): a trial of the Eastern Cooperative Oncology Group. Cancer, 110:759-63.

Etievant C, Barret JM, Kruczynski A, et al. 1998. Vinflunine (20’,20’-difluoro3',4'-dihydrovinorelbine), a novel Vinca alkaloid, which participates in P-glycoprotein (Pgp)-mediated multidrug resistance in vivo and in vitro. Invest New Drugs, 16:3-17.

Etievant C, Kruczynski A, Barret JM, et al. 2001. Markedly diminished drug resistance-inducing properties of vinflunine (20',20'-difluoro-3', 4'-dihydrovinorelbine) relative to vinorelbine, identified in murine and human tumour cells in vivo and in vitro. Cancer Chemother Pharmacol, 48:62-70.

Fabre C, Czaplicki J, Wright M, et al. 2002. Differential binding to the alpha/ beta-tubulin dimer of vinorelbine and vinflunine revealed by nuclear magnetic resonance analyses. Biochem Pharmacol, 64:733-40.

Fahy J, Duflos A, Ribet JP, et al. 1997. Vinca Alkaloids in Superacidic Media: A Method for Creating a New Family of Antitumor Derivatives. $J$ Am Chem Soc, 119:8576-7.

Fahy J, Hellier P, Breillout F, et al. 2008. Vinflunine: discovery and synthesis of a novel microtubule inhibitor. Semin Oncol, 35:S3-S5.

Fechner G, Siener R, Reimann M, et al. 2006. Randomised phase II trial of gemcitabine and paclitaxel second-line chemotherapy in patients with transitional cell carcinoma (AUO Trial AB 20/99). Int J Clin Pract, 60:27-31.

Focan CN, Van Heugen J-C, Kreutz F, et al. 2002. Vinflunine metabolism and disposition in cancer patients. Proc Am Soc Clin Oncol, 21:abstr 495.

Galsky MD, Mironov S, Iasonos A, et al. 2007. Phase II trial of pemetrexed as second-line therapy in patients with metastatic urothelial carcinoma. Invest New Drugs, 25:265-70.

Gebbia V, Testa A, Borsellino N, et al. 1999. Single agent 2', 2'-difluorodeoxycytidine in the treatment of metastatic urothelial carcinoma: a phase II study. Clin Ter, 150:11-5.

Ghoneim MA, el-Mekresh MM, el-Baz MA, et al. 1997. Radical cystectomy for carcinoma of the bladder: critical evaluation of the results in 1,026 cases. J Urol, 158:393-9.

Gout PW, Noble RL, Bruchovsky N, et al. 1984. Vinblastine and vincristine-growth-inhibitory effects correlate with their retention by cultured $\mathrm{Nb}$ 2 node lymphoma cells. Int $J$ Cancer, 34:245-8.

Gregory RK and Smith IE. 2000. Vinorelbine-a clinical review. Br J Cancer, $82: 1907-13$.

Hill BT, Fiebig HH, Waud WR, et al. 1999. Superior in vivo experimental antitumour activity of vinflunine, relative to vinorelbine, in a panel of human tumour xenografts. Eur J Cancer, 35:512-20. 
Hill BT. 2001. Vinflunine, a second generation novel vinca alkaloid with a distinctive pharmacological profile, now in clinical development and prospects for future mitotic blockers. Curr Pharm Des, 7:1199-212.

Hill SA, Lonergan SJ, Denekamp J, et al. 1993. Vinca alkaloids: anti-vascular effects in a murine tumour. Eur J Cancer, 29A:1320-4.

Holwell SE, Hill BT, Bibby MC. 2001. Anti-vascular effects of vinflunine in the MAC 15A transplantable adenocarcinoma model. Br J Cancer, $84: 290-5$.

Hortobagyi GN. 2000. Developments in chemotherapy of breast cancer. Cancer, 88:3073-9.

Jean-Decoster C, Brichese L, Barret JM, et al. 1999. Vinflunine, a new vinca alkaloid: cytotoxicity, cellular accumulation and action on the interphasic and mitotic microtubule cytoskeleton of PtK2 cells. Anticancer Drugs, 10: 37-43

Johnson P, Geldart T, Fumoleau P, et al. 2006. Phase I study of vinflunine administered as a 10-minute infusion on days 1 and 8 every 3 weeks. Invest New Drugs, 24:223-31.

Johnson P, O'Donnell A, Pinel MC, et al. 2001. Phase I Study of Vinflunine Given as a 10 Minute Infusion on Days 1 and 8 Every 3 Weeks in Patients (Pts) with Solid Malignancies. Proc Am Soc Clin Oncol, 20:Abstr 2100.

Johnson SA, Harper P, Hortobagyi GN, et al. 1996. Vinorelbine: an overview. Cancer Treat Rev, 22:127-42.

Joly F, Tchen N, Chevreau C, et al. 2004. Clinical benefit of second line weekly paclitaxel in advanced urothelial carcinoma (AUC): A GETUG phase II study. J Clin Oncol, 22:2004. ASCO Annual Meeting Proceedings (Post-Meeting Edition) 14S (July 15 Supplement):4619.

Jordan MA, Band Horwitz S, Lobert S, et al. 2008. Exploring the Mechanisms of Action of the Novel Microtubule Inhibitor Vinflunine. Semin Oncol, 35:S6-S12.

Jordan MA, Himes RH, Wilson L. 1985. Comparison of the effects of vinblastine, vincristine, vindesine, and vinepidine on microtubule dynamics and cell proliferation in vitro. Cancer Res, 45: 2741-7.

Jordan MA, Thrower D, Wilson L. 1991. Mechanism of inhibition of cell proliferation by Vinca alkaloids. Cancer Res, 51:2212-22.

Kaufman D, Stadler W, Carducci M, et al. 2000. Gemcitabine (G) and Paclitaxel (P) Every Two Weeks (GP2W): A Multicenter Phase II Trial in Locally Advanced or Metastatic Urothelial Cancer (UC). Proc Am Soc Clin Oncol, 19:Abstr 1341.

Kavallaris M, Annereau J-P, Barret J-M. 2008. Potential Mechanisms of Resistance to Microtubule Inhibitors. Semin Oncol, 35:S22-S27.

Khorsand M, Lange J, Feun L, et al. 1997. Phase II trial of oral piritrexim in advanced, previously treated transitional cell cancer of bladder. Invest New Drugs, 15:157-63.

Krege S, Rembrink V, Borgermann C, et al. 2001. Docetaxel and ifosfamide as second line treatment for patients with advanced or metastatic urothelial cancer after failure of platinum chemotherapy: a phase 2 study. J Urol, 165:67-71.

Kruczynski A, Barret JM, Etievant C, et al. 1998a. Antimitotic and tubulininteracting properties of vinflunine, a novel fluorinated vinca alkaloid. Biochem Pharmacol, 55:635-48.

Kruczynski A, Colpaert F, Tarayre JP, et al. 1998b. Preclinical in vivo antitumor activity of vinflunine, a novel fluorinated vinca alkaloid. Cancer Chemother Pharmacol, 41:437-47.

Kruczynski A, Etievant C, Perrin D, et al. 2002. Characterization of cell death induced by vinflunine, the most recent Vinca alkaloid in clinical development. Br J Cancer, 86:143-50.

Le Chevalier T, Brisgand D, Douillard JY, et al. 1994. Randomized study of vinorelbine and cisplatin versus vindesine and cisplatin versus vinorelbine alone in advanced non-small-cell lung cancer: results of a European multicenter trial including 612 patients. J Clin Oncol, 12:360-7.

Lemarie E, Bennouna J, Grossi F, et al. 2005. Vinflunine (VFL) in combination with gemcitabine (GEM) for treatment of advanced non-small cell lung cancer (NSCLC) in chemonaive patients (pts): Preliminary results of the phase I and pharmacokinetic study. J Clin Oncol, 23; 2005. ASCO Annual Meeting Proceedings (Post-Meeting Edition) 16S (July 15 Suppl):7266.
Lin CC, Hsu CH, Huang CY, et al. 2007. Gemcitabine and ifosfamide as a second-line treatment for cisplatin-refractory metastatic urothelial carcinoma: a phase II study. Anticancer Drugs, 18:487-91.

Lobert S and Puozzo C. 2008. Pharmacokinetics, metabolites, and preclinical safety of vinflunine. Semin Oncol, 35:S28-S33.

Lobert S, Ingram JW, Hill BT, et al. 1998. A comparison of thermodynamic parameters for vinorelbine- and vinflunine-induced tubulin self-association by sedimentation velocity. Mol Pharmacol, 53:908-15.

Loehrer PJ Sr, Einhorn LH, Elson PJ, et al. 1992. A randomized comparison of cisplatin alone or in combination with methotrexate, vinblastine, and doxorubicin in patients with metastatic urothelial carcinoma: a cooperative group study. J Clin Oncol, 10:1066-73.

Logothetis CJ, Dieringer P, Ellerhorst J, et al. 1992. A 61\% response rate with 5-fluorouracil, interferon-a $2 \mathrm{~b}$ and cisplatin in metastatic chemotherapy refractory transitional cell carcinoma. Proc Am Assoc Cancer Res, 33:221.

Lorusso V, Pollera CF, Antimi M, et al. 1998. A phase II study of gemcitabine in patients with transitional cell carcinoma of the urinary tract previously treated with platinum. Italian Co-operative Group on Bladder Cancer. Eur J Cancer, 34:1208-12.

McCaffrey JA, Hilton S, Mazumdar M, et al. 1997. Phase II trial of docetaxel in patients with advanced or metastatic transitional-cell carcinoma. J Clin Oncol, 15:1853-7.

Meluch AA, Greco FA, Burris HA 3rd, et al. 2001. Paclitaxel and gemcitabine chemotherapy for advanced transitional-cell carcinoma of the urothelial tract: a phase II trial of the Minnie pearl cancer research network. J Clin Oncol, 19:3018-24.

Moore M, Winquist E, Vokes E, et al. 2003. Phase II study of oxaliplatin in patients with inoperable, locally advanced or metastatic transitional cell carcinoma of the urothelial tract (TCC) who have received prior chemotherapy. Proc Am Soc Clin Oncol, 22:Abstr 1638.

Ngan VK, Bellman K, Hill BT, et al. 2001. Mechanism of mitotic block and inhibition of cell proliferation by the semisynthetic Vinca alkaloids vinorelbine and its newer derivative vinflunine. Mol Pharmacol, 60:225-32.

Ngan VK, Bellman K, Panda D, et al. 2000. Novel actions of the antitumor drugs vinflunine and vinorelbine on microtubules. Cancer Res, 60:5045-51

Pagliaro LC, Millikan RE, Tu SM, et al. 2002. Cisplatin, gemcitabine, and ifosfamide as weekly therapy: a feasibility and phase II study of salvage treatment for advanced transitional-cell carcinoma. J Clin Oncol, 20:2965-70.

Papamichael D, Gallagher CJ, Oliver RT, et al. 1997. Phase II study of paclitaxel in pretreated patients with locally advanced/metastatic cancer of the bladder and ureter. Br J Cancer, 75:606-7.

Paridaens R, Wildiers H, Dalenc F, et al. 2007. Vinflunine in combination with trastuzumab for treatment of HER2-positive metastatic breast cancer. J Clin Oncol, 2007 ASCO Annual Meeting Proceedings Part I. Vol 25, No. 18S (June 20 Supplement), 2007: 1058.

Parkin DM, Bray F, Ferlay J, et al. 2005. Global cancer statistics, 2002. CA Cancer J Clin, 55: 74-108.

Paule B, Saliba F, Gil-Delgado M, et al. 2007. Phase I and pharmacokinetic (PK) dose-adjusted study of IV vinflunine (VFL) in cancer patients with liver dysfunction (LD): Pharmacokinetic results. J Clin Oncol, 25; 2007. ASCO Annual Meeting Proceedings 25(18S) (June 20 Suppl): 2523.

Pourroy B, Carre M, Honore S, et al. 2004. Low concentrations of vinflunine induce apoptosis in human SK-N-SH neuroblastoma cells through a postmitotic G1 arrest and a mitochondrial pathway. Mol Pharmacol, 66:580-91.

Pronzato P, Vigani A, Pensa F, et al. 1997. Second line chemotherapy with ifosfamide as outpatient treatment for advanced bladder cancer. Am J Clin Oncol, 20: 519-21.

Puozzo C, Vermorken JB, Bauer J, et al. 2001. A Phase I Pharmacokinetic Study of Vinflunine Given on a Weekly Schedule. Proc Am Soc Clin Oncol, 20:Abstr 2107. 
Ramlau R, Souquet P-J, Sun X, et al. 2004. Results of a phase I/II and pharmacokinetic study of vinflunine (VFL) in combination with cisplatin (CDDP) for primary treatment of advanced non-small cell lung cancer (NSCLC). J Clin Oncol, 22:2004 ASCO Annual Meeting Proceedings (Post-Meeting Edition) (14S) (July 15 Suppl): 7110.

Rosenberg JE, Carroll PR, Small EJ. 2005. Update on chemotherapy for advanced bladder cancer. $J$ Urol, 174:14-20.

Roth BJ, Manola J, Dreicer R, et al. 2002. Piritrexim in advanced, refractory carcinoma of the urothelium (E3896): a phase II trial of the Eastern Cooperative Oncology Group. Invest New Drugs, 20:425-9.

Saliba F, Paule B, Adam R, et al. 2007. Vinflunine in patients with advanced unresectable hepatocellular carcinoma and liver impairment. $J$ Clin Oncol, 25; 2007 ASCO Annual Meeting Proceedings (18S) (June 20 Suppl): 15023 .

Shah U, Sanoff HK, O'Neil BH, et al. 2008. A phase I trial of pemetrexed and vinflunine (VFL). J Clin Oncol, 26(May 20 Suppl):Abstr 13508.

Singer WDandHimes RH. 1992. Cellular uptake and tubulin binding properties of four Vinca alkaloids. Biochem Pharmacol, 43:545-51.

Sridhar SS, Stadler W, Le L, et al. 2005. Phase 2 study of Bortezomib in Advanced or Metastatic Urothelial Cancer. A trial of the Princess Margaret Hospital [PMH] Phase II Consortium. J Clin Oncol, 23; ASCO Annual Meeting Proceedings (16S, Part I of II)(June 1 Suppl): 4677.

Stein JP, Lieskovsky G, Cote R, et al. 2001. Radical cystectomy in the treatment of invasive bladder cancer: long-term results in 1,054 patients. J Clin Oncol, 19:666-75.

Stein JP. 2006. Improving outcomes with radical cystectomy for high-grade invasive bladder cancer. World J Urol, 24:509-16.

Sternberg CN and Vogelzang NJ. 2003. Gemcitabine, paclitaxel, pemetrexed and other newer agents in urothelial and kidney cancers. Crit Rev Oncol Hematol, 46(Suppl):S105-15.

Sternberg CN, Calabro F, Pizzocaro G, et al. 2001. Chemotherapy with an every-2-week regimen of gemcitabine and paclitaxel in patients with transitional cell carcinoma who have received prior cisplatin-based therapy. Cancer, 92:2993-8.

Sternberg CN, Yagoda A, Scher HI, et al. 1988. M-VAC (methotrexate, vinblastine, doxorubicin and cisplatin) for advanced transitional cell carcinoma of the urothelium. J Urol, 139:461-9.

Sweeney CJ, Roth BJ, Kabbinavar FF, et al. 2006. Phase II study of pemetrexed for second-line treatment of transitional cell cancer of the urothelium. J Clin Oncol, 24:3451-7.

Sweeney CJ, Williams SD, Finch DE, et al. 1999. A Phase II study of paclitaxel and ifosfamide for patients with advanced refractory carcinoma of the urothelium. Cancer, 86:514-8.
Tourani J, Pinel M, Planchard D, et al. 2005. Phase I and pharmacokinetic study of Vinflunine (VFL) in combination with carboplatin (CBDCA) for treatment of advanced non-small cell lung cancer (NSCLC) in chemonaive patients (pts): Final results. J Clin Oncol, 23; ASCO Annual Meeting Proceedings (16S, Part I of II) (June 1 Suppl): 7271.

Tu SM, Hossan E, Amato R, et al. 1995. Paclitaxel, cisplatin and methotrexate combination chemotherapy is active in the treatment of refractory urothelial malignancies. J Urol, 154:1719-22.

Vaishampayan UN, Faulkner JR, Small EJ, et al. 2005. Phase II trial of carboplatin and paclitaxel in cisplatin-pretreated advanced transitional cell carcinoma: a Southwest Oncology Group study. Cancer, 104:1627-32.

Vaughn DJ, Broome CM, Hussain M, et al. 2002. Phase II trial of weekly paclitaxel in patients with previously treated advanced urothelial cancer $J$ Clin Oncol, 20:937-40.

Vaughn DJ, Srinivas S, Petrylak DP, et al. 2008. Vinflunine (VFL) in patients with platinum-refractory transitional cell carcinoma of the urothelium (TCCU): results of a large phase 2 study. 2008. ASCO Genitourinary Cancers Symposium, Abstr 316.

Vermorken JB, Stupp R, Nguyen L, et al. 2003. Phase I Study of IV vinflunine given on a weekly schedule in previously untreated patients (pts) with advanced solid tumors. Proc Am Soc Clin Oncol, 22:Abstr 887.

von der Maase H, Hansen SW, Roberts JT, et al. 2000. Gemcitabine and cisplatin versus methotrexate, vinblastine, doxorubicin, and cisplatin in advanced or metastatic bladder cancer: results of a large, randomized, multinational, multicenter, phase III study. J Clin Oncol, 18:3068-77.

Winquist E, Kirchner TS, Segal R, et al. 2004. Neoadjuvant chemotherapy for transitional cell carcinoma of the bladder: a systematic review and meta-analysis. J Urol, 171:561-9.

Witte RS, Elson P, Bono B, et al. 1997. Eastern Cooperative Oncology Group phase II trial of ifosfamide in the treatment of previously treated advanced urothelial carcinoma. J Clin Oncol, 15:589-93.

Witte RS, Manola J, Burch PA, et al. 1998. Topotecan in previously treated advanced urothelial carcinoma: an ECOG phase II trial. Invest New Drugs, 16:191-5.

Wülfing C, Machiels J, Richel D, et al. 2005. A single arm, multicenter, open-label, ph II study of lapatinib as $2 \mathrm{~L}$ treatment of pts with locally advanced/metastatic transitional cell carcinoma (TCC) of the urothelial tract. J Clin Oncol, 23; ASCO Annual Meeting Proceedings. (16S, Part I of II) (June 1 Suppl):4594.

Zorza G, Johnson P, Judson I, et al. 2001. A Phase I Pharmacokinetic Study of Vinflunine Given on Days 1 and 8 Every 3 Weeks. Proc Am Soc Clin Oncol, 20:Abstr 2070. 
